\title{
Combined Wind Speed and Angle Control in a Virtual Environment Using a Static Observer
}

\author{
Sandip D. Kulkarni, Mark A. Minor, Eric R. Pardyjak and John M. Hollerbach
}

\begin{abstract}
This paper develops a static observer for estimating wind speed in order to control wind speed and angle control at a user position in a submersive virtual environment. Addition of wind display evolves the Treadport Virtual environment into a highly immersive virtual environment called Treadport Active Wind Tunnel (TPAWT). Experiments on a scaled model of the TPAWT show that headwind flow stream diverges at the user. Pitot tube sensors placed at a particular region of converged flow in the scaled TPAWT provide measurements with lower noise. Open loop experiments on a scaled model of TPAWT show that there exists a relationship between speed measured at this region of converged flow and the wind speed at the user position. Using this relationship, the wind speed at the user can be estimated. We use this relation and combine previously used speed and angle controllers based upon the small gain theorem with a dynamic extension and conditional angular rate-switching control. Finally, we simultaneously control wind speed and headwind angle.
\end{abstract}

\section{INTRODUCTION}

$\mathrm{T}$ HIS paper develops a static observer for estimating the wind flow speed in order to simultaneously control wind speed and angle at a user in the Treadport Virtual Environment, Fig. 1. The Treadport Virtual Environment [1] already has a locomotion interface, which allows the user to walk through the virtual environment while viewing realistic rendering on a $180^{\circ} \mathrm{CAVE}-$ like display provided by visual screens on the front and the sides. Current works [24] on augmenting the haptic interface to the original Treadport Virtual Environment [1], termed TreadPort Active Wind Tunnel (TPAWT) involve creating wind flow at the user. A haptic interface improves the sense of immersion for the user [5]. The side screens of the TPAWT guide the air flowing through side vents, Fig. 2, and provide passive control. The flow merges and separates at the front screen. An air column flows between two counter-rotating vortices

Manuscript received February 22, 2008. This work was supported in part by the National Science Foundation under Grant No 428856 (sponsor and financial support acknowledgment goes here)

S.D. Kulkarni is with the Department of Mechanical Engineering, University of Utah, Salt Lake City, Utah, 84112, and USA. (sandip.kulkarni@utah.edu)

M.A. Minor is with the Department of Mechanical Engineering, University of Utah, Salt Lake City, Utah, 84112, USA (phone: 801-5877771; fax: 801-585-9826; minor@mech.utah.edu).

E.R. Pardyjak is with the Department of Mechanical Engineering, University of Utah, Salt Lake City, Utah, 84112, USA.

J.M. Hollerbach is with the School of Computing, University of Utah, Salt Lake City, Utah, 84112, USA towards the user. In [4], a return plenum shown in Fig. 2, is augmented to the scaled model to stabilize wind flow and allow input-output control of wind angle using a version of Small Gain Theorem (SGT) [6] and dynamic extension in combination with a conditional angular rate switching control.

This paper contributes in developing a static observer to estimate the speed at the user, by measuring speed at the return plenum. We incorporate the static observer in the speed controller developed in [3], combine it with the headwind angle controller from [4] and achieve simultaneous wind speed and headwind angle control at the user. From [4] and Fig. 2, observe that the headwind flow diverges at the user. There is a high variance in the speed measurement when flow diverges. Since wind flow converges at the outlet of return plenum, Fig. 2, the pitot

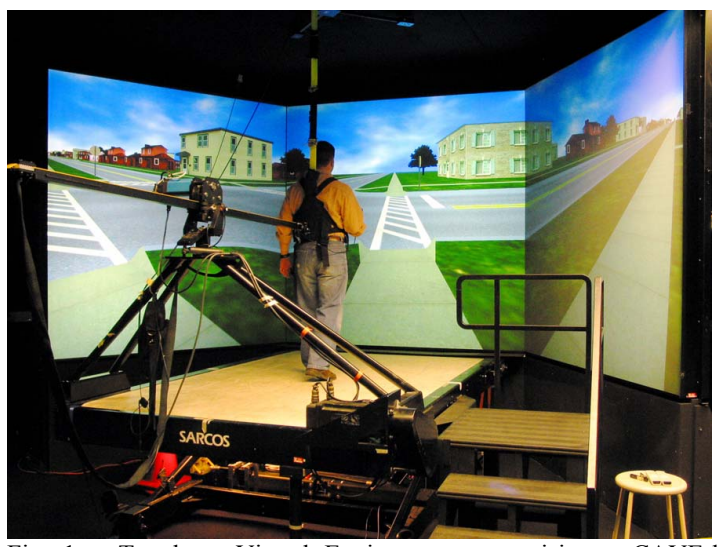

Fig. 1. . Treadport Virtual Environment comprising a CAVE-like visual display and locomotion interface

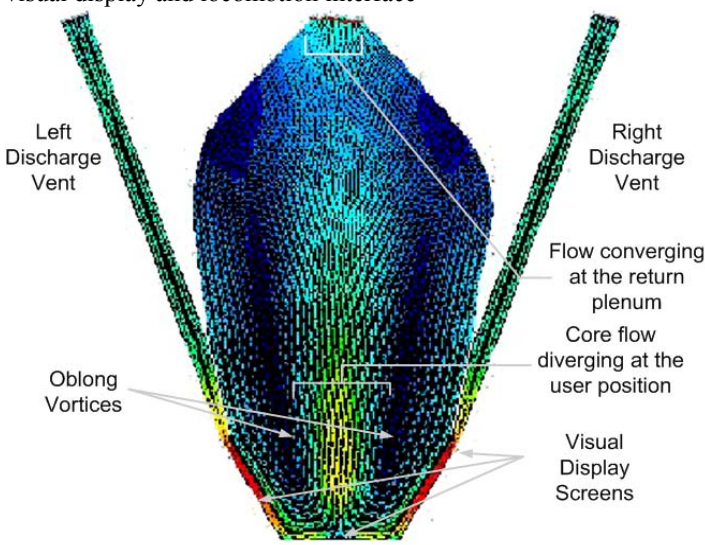

Fig. 2 Velocity vector plot of the input-output characterization using FLUENT 
tube measurements have lower noise in this region. Furthermore, experiments show that the wind speed at the outlet of return plenum is statically related to the wind speed at the user.

The paper is organized as follows. Sect. II compares this research to and follows on the prior work. The flow is characterized in Sect. III. The previous speed and angle controllers are combined and modified using a static observer in Sect IV and evaluated in Sect V. Future work is discussed in Sect VI. Sect. VII concludes the paper.

\section{BACKGROUND}

Controlling wind flow in TPAWT is a problem of active flow control. Fluid flow is an infinite dimensional system. Thus, control of fluid flow has been studied using reduced order modeling techniques such as Proper Orthogonal Decomposition (POD) [7, 8], Approximate Inertial Manifolds (AIM) [9], and Eigenmode [10] analysis. Model decomposition methods reduce the DOF to a certain extent, but there still remains a vast gap between the DOF required for real-time active-control of wind flow and the DOF after model decomposition. Moreover change in basis with varying flow patterns must be accounted for and is computationally expensive. Instead, our method focuses on stabilizing the flow using a combination of an output feedback algorithm based upon the SGT and a conditional angular rate-switching feedback control aided with a static observer for estimation of flow speed.

Accurate sensor placement is necessary for maximizing the performance of a controller and allowing effective use of sensors. In [11] it is shown that pitot static tube is a feasible choice for the real-time measurement of the wind speed in the scaled model of TPAWT. The pitot static tube measures wind speed while a single vane attached to the probe rotates about its longitudinal axis. According to [12], the measurements from the pitot static probe are insensitive to angle of attack from $-2^{\circ}$ to $12^{\circ}$. The vane aligns the probe with the direction of wind when the angle of attack is outside the above range. In [11], pressure transducer calibration coefficients account for the sensor nonlinearities.

Rather than a theoretical development for sensor placement as explained in [13], we use an experimentally derived static observer for estimating flow at the user position. As far as we know, our application of dynamic extension and the small gain theorem combined with the static observer approach and conditional angular rateswitching control is a novel flow control application. Furthermore, we provide a method for estimating wind speed at the user that will allow more advanced configurations to make less intrusive sensors for virtual environments.

\section{FLOW CHARACTERIZATION}

The stream function plot generated by unsteady FLUENT simulations shown in Fig. 2 indicates the development of a stable head wind at the user. Ideally, if left and right vent velocities, $v_{L}$ and $v_{R}$, respectively, are equal then the vortices are symmetric and the wind angle is $\theta=0^{\circ}$.

Firstly, we develop nominal input-output mapping between the ratios of vent velocities $\mathrm{v}_{\mathrm{L}}$ and $\mathrm{v}_{\mathrm{R}}, \mathrm{v}_{\mathrm{L}} / \mathrm{v}_{\mathrm{R}}$, and the heading angle $\theta$ from unsteady FLUENT simulations. At ratios far from 1, the vortices shift the core flow considerably and the core flow is again attracted to one of the stable equilibrium at $\theta= \pm 60^{\circ}$ [2] Hence we consider only the vent velocity ratios in the range from 0.75 to 1.25 . A series of simulations were conducted to sweep this range. At each vent velocity ratio, the flow was allowed to reach steady state and the wind angle was measured to produce Fig. 3. Wind speed at a given heading angle is then mapped with the vent outlet velocities for a fixed vent velocity ratio of $v_{L} / v_{R}=1.00$. As Fig. 4 indicates, we also develop a map between the total inlet speed, $v_{L}+v_{R}$, and the speed at the user position. We see that this trend is also linear and generally true for various values of fixed $v_{R}$.

Using the results from Fig. 3 and Fig. 4, linear least square estimation is performed to estimate the geometric Jacobian, $\mathrm{J}$ given in (1) below.

$$
\delta y=J \delta u \text {. }
$$

The slope of this line is essentially the Jacobian. The Jacobian for all of the points is obtained by taking a generalized inverse of the input, as

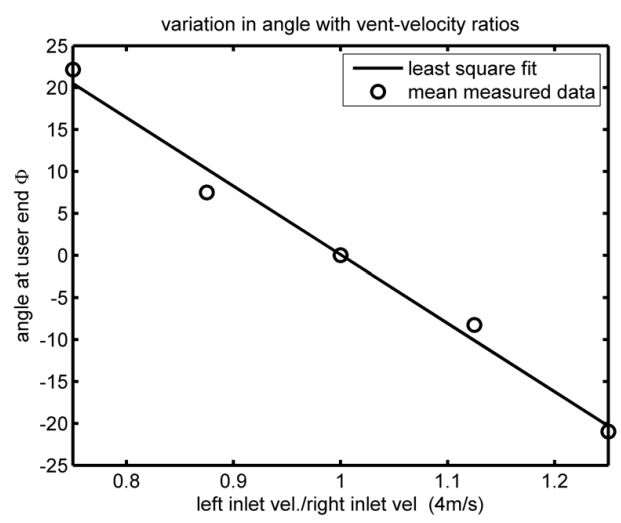

Fig. 3 Wind angle versus vent velocity ratio $u$, based upon unsteady CFD simulations with the return plenum.

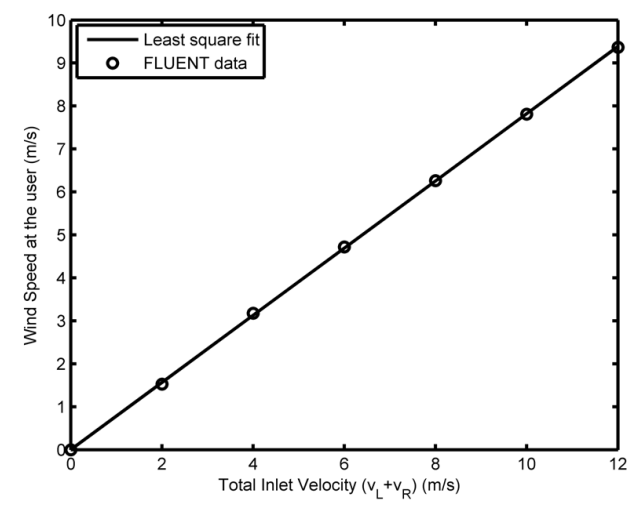

Fig. 4. Wind speed at user given $v_{L} / v_{R}=1.00$ as total vent velocity $\mathrm{v}_{\mathrm{L}}+\mathrm{v}_{\mathrm{R}}$ is increased. 


$$
J=\delta y \delta u^{-1}
$$

where $\delta y$ is the change in wind angle or speed measured by sensors $\delta u$ is change in the ratio of wind velocities at the vents or the sum of vent velocities $\left(\mathrm{v}_{\mathrm{L}}+\mathrm{v}_{\mathrm{R}}\right)$. Thus, the geometric Jacobian is $J_{\theta} \approx-85^{\circ}$ for wind angle or $J_{v} \approx 0.78$ for wind speed.

\section{CONTROLler DEVEloPMENT}

In this paper, we combine the input-output feedback controller for wind speed developed in [3] and the feedback controller for wind angle developed in [4] as shown in Fig. 5. We revisit the controller development in a generalized manner for the sake of completeness and introduce a static observer to estimate wind speed.

\section{A. Dynamic Extension and Small Gain Theorem}

The error states are defined as $e=y-y_{R}$ where $y_{R}$ is the desired output (desired wind speed or angle) and $y$ is the actual output wind speed at the user or the actual wind angle. Taking the time derivative we have,

$$
\dot{e}=\frac{\partial e}{\partial t}=\frac{\partial y}{\partial t}-\frac{\partial y_{R}}{\partial t} .
$$

Applying the chain rule to (3),

$$
\dot{e}=\frac{\partial y}{\partial u} \frac{\partial u}{\partial t}-\frac{\partial y_{R}}{\partial t},
$$

and substituting the geometric Jacobian, $J=\frac{\partial y}{\partial u}$, we then have,

$$
\dot{e}=J \dot{u}-\frac{\partial y_{R}}{\partial t}=g(e) .
$$

Assigning $g(e)=-K e$, where $K=K^{\mathrm{T}}>0$ is Hurwitz, we can then solve for the desired control inputs,

$$
\dot{u}=J^{-1}\left[-K e+\dot{y}_{r}\right]
$$

in a fashion similar to backstepping. Assuming regulation only, the resulting control system is then expressed as,

$$
\begin{aligned}
& \dot{e}=-K e=K\left(y_{r}-y\right) . \\
& \dot{u}=-J^{-1} K e
\end{aligned} .
$$

Note that the control command $u$ now appears as a state variable. We have applied a dynamic extension to the input. This allows an increase in the relative degree of the system. Unique to this controller is that only the Jacobian $J$ is required for a given operating point. This simplicity allows easy implementation of this controller in real-time and requires minimal memory.

The feedback system (6) can be represented in the form of interconnected systems for combined wind speed and angle control as shown in Fig. 5. Table 1 shows the notations for the two controllers:

Application of the Small Gain Theorem requires that the

TABLE 1:

NOTATIONS FOR THE INPUTS, OUTPUTS, GAINS AND THE TPAWT MAPS FOR THE TWO CONTROLLERS

\begin{tabular}{|l|l|l|}
\hline & \multicolumn{1}{|c|}{ Speed Control } & Angle Control \\
\hline Input $u$ & $\begin{array}{r}u_{v}=v_{L}+v_{R} \\
=-\int J_{v}^{-1} \cdot K_{v} e d t\end{array}$ & $\begin{array}{r}u_{\theta}=v_{L} / v_{R} \\
=-\int J_{\theta}^{-1} \cdot K_{\theta} e d t\end{array}$ \\
& & \\
\hline Output $y$ & Wind Speed $y$ & Wind Angle $\theta$ \\
\hline Gain $K$ & Gain $K_{v}$ & Gain $K_{\theta}$ \\
\hline Map & Slope $J_{v}$ & Slope $J_{\theta}$ \\
\hline
\end{tabular}

aforementioned subsystems are finite gain $L$-stable as described by,

$$
\begin{gathered}
\|u\| \leq \gamma_{1}\|e\|_{L}+\beta_{1} \\
\|y\| \leq \gamma_{2}\|u\|_{L}+\beta_{2} .
\end{gathered}
$$

where $\gamma_{1}, \gamma_{2}, \beta_{1}$, and $\beta_{2}$ must all be finite values. Discretizing the subsystems for simplicity during experiments and taking $L$ norms we have,

$$
\begin{gathered}
\left\|u_{k}\right\|_{L} \leq\left\|J^{-1} K\right\|_{L}\left\|e_{k}\right\|_{L} \Delta t+u_{k-1} \\
\left\|y_{k}\right\|_{L} \leq\|J\|_{L}\left\|u_{k}\right\|_{L} \Delta t .
\end{gathered}
$$

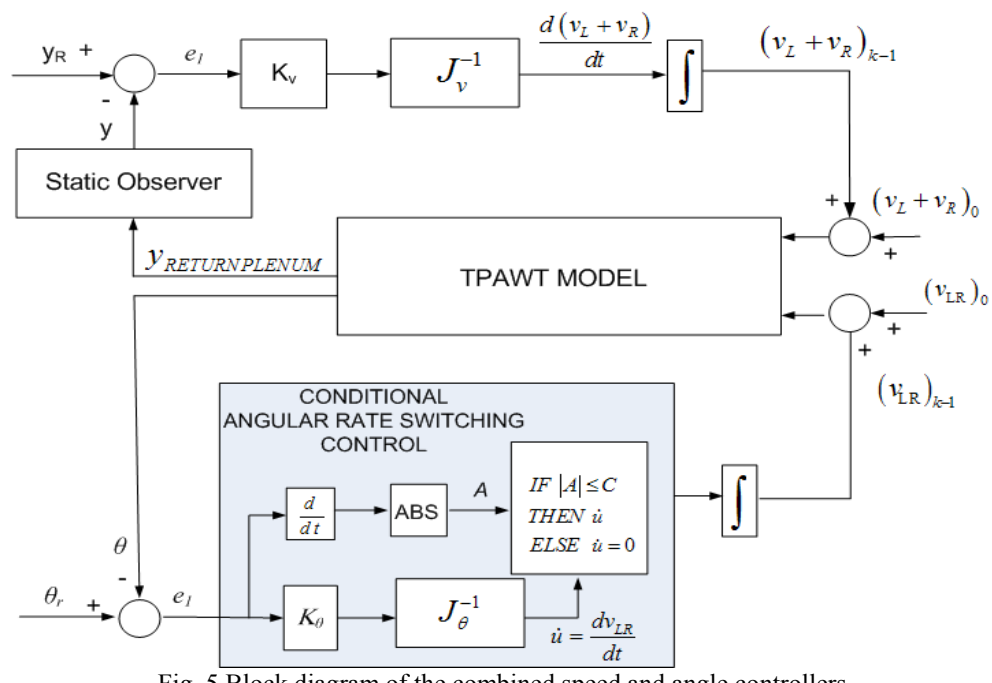

Fig. 5 Block diagram of the combined speed and angle controllers 
From (7)-(10) we have,

$$
\begin{gathered}
\gamma_{1}=\left\|J^{-1}\right\|_{L} \cdot\|K\|_{L} \cdot \Delta t, \\
\gamma_{2}=\|J\|_{L}, \\
\beta_{1}=u_{k-1} \quad \beta_{2}=0
\end{gathered}
$$

Considering a discrete system with fixed sampling rates, it is important to note that $\gamma_{1}$ and $\gamma_{2}$ do not change. Furthermore, the SGT requires that $\gamma_{1} \gamma_{2}<1$ [6]. Given (11), the feedback connection is finite-gain $L$-stable by the SGT if,

$$
\|K\|_{L} \Delta t<1 \text {. }
$$

\section{B. Conditional Angular Rate-Switching Controller}

$(C A R S)$

In the case of wind angle control, when the controller attempts to regulate wind angle, design asymmetry and process delays in the system coupled with nonlinear energy exchange between the vortices results in rapid switching of wind angle.

In order to resolve the rapid nonlinear oscillations of wind angle, the Conditional Angular Rate Switching controller[4], shown in Fig. 5 is used. If the rate of change of wind angle, $A=|\dot{e}|$, exceeds a bound $\mathrm{C}$, the controller is paused by setting the input to the integrator to zero, and thus the vent velocity ratio is maintained at a constant value. The

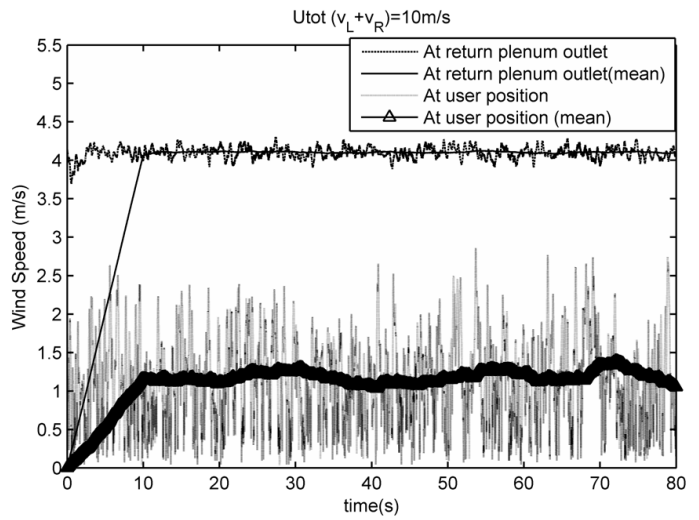

Fig. 6 Flow speeds measured at the user position and the outlet of the return plenum for total inlet speed $\left(\mathrm{v}_{\mathrm{L}}+\mathrm{v}_{\mathrm{R}}\right)=10 \mathrm{~m} / \mathrm{s}$

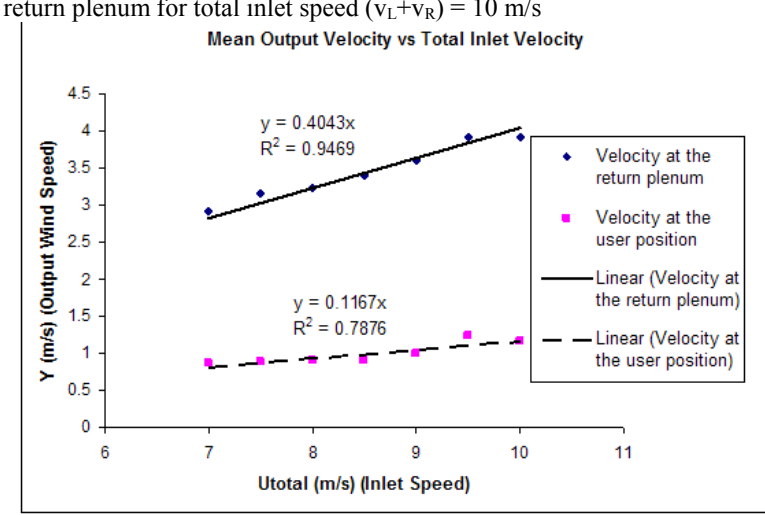

Fig. 7 Output wind speeds measured at the user position and the outlet of the return plenum for various total inlet speeds $\left(\mathrm{v}_{\mathrm{L}}+\mathrm{v}_{\mathrm{R}}\right)$ momentum gradually transfers between the vortices and the wind angle variations are retarded. Once $A \leq C$, the controller is re-engaged and the wind angle is regulated by the output feedback controller.

\section{Sensor measurements and static observer}

The computational fluid dynamic simulation in Fig. 2 show that the wind flow diverges when the flow is head-on at the user position. We measure wind speed at the user position where the flow diverges and the wind speed at the outlet of the return plenum where the flow converges before leaving the test section of TPAWT. The pitot tube measurements at the user have lot of noise in comparison to the measurements at the outlet of the return plenum as shown in Fig. 6. Moreover the divergence of flow reduces the speed at the user position. The standard deviation of the speed at the user position is $\sim 0.6$ while that at the outlet of the return plenum is $\sim 0.07$ for 10 trials and repeated over .

Table 2 displays the wind speed at the user position ( $\mathrm{Y}_{\text {user }}$ and at the outlet of the return plenum $\left(\mathrm{Y}_{\text {outlet }}\right)$ measured for total inlet speed $\left(\mathrm{v}_{\mathrm{L}}+\mathrm{v}_{\mathrm{R}}\right)$ ranging from $7 \mathrm{~m} / \mathrm{s}$ to $10 \mathrm{~m} / \mathrm{s}$. Note that the vent velocity ratio $\left(\mathrm{v}_{\mathrm{L}} / \mathrm{v}_{\mathrm{R}}\right)$ is fixed to 1 and an open loop control is implemented. The fourth column of Table 2 shows that the ratio of speed at the user to the speed at the outlet of return plenum $\left(\mathrm{Y}_{\text {user }} / \mathrm{Y}_{\text {outlet }}\right)$ is $\sim 0.3$. This ratio can be used to relate the two sensor measurements. Fig. 7 shows the input-output maps of inlet speed to wind speed at the outlet of return plenum and the user. The least square regression give a linear fit for a set of 7 experiments. We use the speed ratio $\left(\mathrm{Y}_{\text {user }} / \mathrm{Y}_{\text {outlet }}\right)$, as a static observer and use the wind speed at the outlet of the return plenum to estimate the speed at the user position. This estimated speed is used as a feedback for the wind speed control shown in Fig. 5.

\begin{tabular}{|c|c|c|c|c|}
\hline $\mathrm{U}_{\text {total }}$ & $\begin{array}{c}Y_{\text {outlet }} \\
\text { (mean) }\end{array}$ & $\begin{array}{c}\mathrm{Y}_{\text {user }} \\
\text { (mean) }\end{array}$ & $\begin{array}{c}\mathrm{Y}_{\text {user }} / \mathrm{Y}_{\text {outlet }} \\
\text { (Speed Ratio) }\end{array}$ & $\begin{array}{c}\text { Vane Angle } \\
\left(\sim 0^{\circ}\right)\end{array}$ \\
\hline 7.0 & 2.91 & 0.86 & 0.3 & -4.28 \\
\hline 7.5 & 3.15 & 0.88 & 0.28 & -2.40 \\
\hline 8.0 & 3.23 & 0.90 & 0.28 & -2.66 \\
\hline 8.5 & 3.40 & 0.90 & 0.26 & 1.1 \\
\hline 9.0 & 3.59 & 1.00 & 0.28 & -3.85 \\
\hline 9.5 & 3.91 & 1.23 & 0.31 & -6.36 \\
\hline 10.0 & 4.1 & 1.21 & 0.30 & -7.28 \\
\hline
\end{tabular}
This eliminates the noise without introducing delay into the system.

TABLE 2

\section{EXPERIMENTAL EVALUATION}

\section{A. Scaled Test Bed}

The scale model, Fig. 8, incorporates actuated valves and a number of velocity sensors in order to facilitate real-time flow control. It is composed of a blower, a main plenum, butterfly throttling valves, ducting, a test section, return plenum and settling plenum. Throttling valves are mounted 
on the side of the plenum and are actuated by geared $(66: 1)$ Maxon DC motors (20 W).

Ducting connects the valves to the TPAWT test section as in [3]. The inlet vents are mounted symmetrically at an angle $\Phi$ with the test section walls. Experiments show that the flow angle at the user in insensitive to this angle $\Phi$. The return plenum is provided to stabilize the flow with the help of a negative pressure. The test section is comprised of Lexan (side walls, ceiling) and wood (floor). In order to approximate 2D flow, the gap height between floor and ceiling was reduced to $13 \mathrm{~cm}$ throughout the test section.

The TPAWT test section has pitot tubes at each of the inlet ducts to determine vent velocities. A pitot-vane sensor mounted at the user position determines wind speed and direction at the user. The vane was designed to orient the pitot tube along the streamlines of the flow. Dwyer 607-21 Differential Pressure Transmitters with a $250 \mathrm{~ms}$ response time were used to measure pitot tube pressure. Potentiometers measure valve angles and pitot-vane angle.

A dSpace 1103 Controller Board operating at a $1 \mathrm{kHz}$ sampling rate is used to control the experimental apparatus from Simulink using the Real Time Workshop toolbox. Vent velocity is regulated by PI control loops that regulate valve angles. Blower speed (e.g. fan frequency) is manually regulated before the experiments to assure that sufficient plenum pressure is available to supply adequate flow through the vents.

\section{B. Results and Discussion}

Fig. 9 and Fig. 10 show the controlled wind speed and the wind angle at the user. By estimating the wind speed at the user position, we are avoiding the feedback of the noisy transient part of the speed response at the user. This

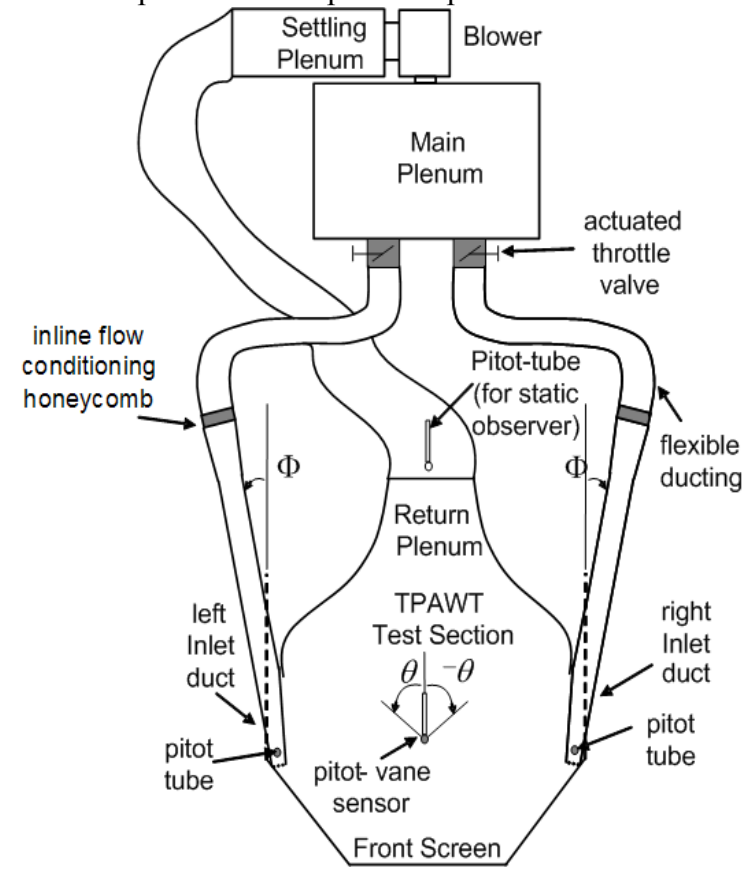

Fig. 8 Schematic for Quarter Scaled 2D Model of Treadport Active Wind Tunnel improves performance of the wind speed response while maintaining the wind angle control. Note that the control of wind speed at the user along with wind angle improves the perception of wind by the user.

In Fig. 10, the delay indicates that the vortices take almost $40 \mathrm{~s}$ to get energized and push the core flow towards the user. The four principles of vortex motion: a) space filling, b) self-organizing c) parity adjusting and d) spectral refining as described in [14] along with time delay keep the pitot vane static for $40 \mathrm{~s}$. The vent velocity ratio $v_{L} / v_{R}$ is the controlled input as shown in Table 1, and keeps increasing until it reaches a threshold. At this moment, there is nonlinear exchange of energy between the vortices that shifts the flow abruptly. Here the rate of change of wind angle, A, has exceeded the bound $\mathrm{C}$ as detailed in IV.B and the CARS controller sets $\dot{u}=0$ pausing the core flow at headwind configuration. When $\mathrm{A}<\mathrm{C}$, the output feedback controller is again reactivated. The wind angle control is detailed in [4].

According to Fig. 9, the speed at the user position is 1.2 $\mathrm{m} / \mathrm{s}$. Since the head-on flow diverges at the user, the speed is naturally lower in comparison to the inlet and outlet speeds. In our ongoing research we are providing a slit in the floor of the front screen of the TPAWT. Pressure difference between the atmosphere and the TPAWT will

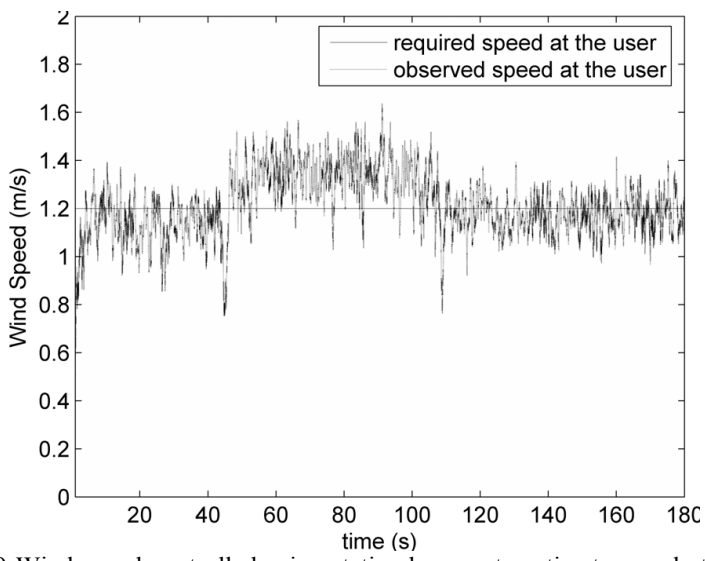

Fig. 9 Wind speed controlled using static observer to estimate speed at the user

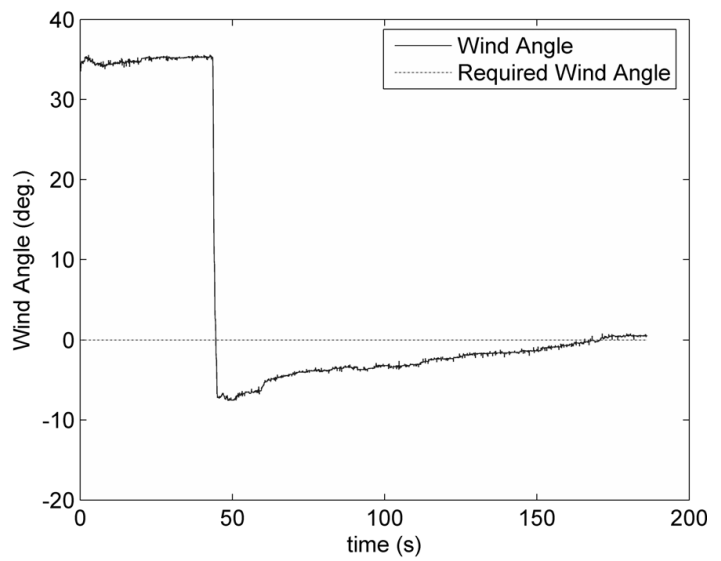

Fig. 10 Wind angle control using input output feedback control and CARS controller combined with speed control 
cause air to flow through the slit and merge with the core flow. This will compensate for the loss of mass absorbed around the vortices due to flow divergence.

The static observer plays a major role in estimating the wind speed at the user without intruding the user environment. In order to verify if the relationship between the wind speed at the user and the outlet of return plenum is static for other wind angles, we conduct several experiments for a steady wind angle of $\sim 30^{\circ}$. The results tabulated in Table 3, show that speed ratio $\left(\mathrm{Y}_{\text {user }} / \mathrm{Y}_{\text {outlet }}\right)$ is consistently about 0.6. Observe that the speed ratio has increased from 0.3 to 0.6 as the wind angle has increased from $\sim 0^{\circ}$ to $\sim 30^{\circ}$.

\section{FUTURE WORK}

After introducing a slit in the floor of the front screen we will test the speed and angle control for a variety of wind angles. The controllers will then be validated using the full scale Treadport system. In the full-scale system, we will use methods similar to the development of static observer, in order to make the sensor measurements non-intrusive to the user environment. Flow direction probes and hot wire anemometers are some of the alternatives for estimating wind speed as well as angle at the user. Further research and analysis will be required to map the relationship between wind speeds and angles observed at different positions of the sensors as indicated by Table 2 and Table 3. This method of estimating sensor data can be useful in particular applications where sensors cannot be placed all the time.

Since we do not have an accurate model of the system, we will also be applying reinforcement learning methods such as temporal differencing[15] and policy gradient methods[16] to improve performance of the wind speed and angle response. We will be developing policies from FLUENT simulations as well as experimental maps.

\section{CONCLUSION}

In this paper, we achieve simultaneous wind speed and headwind angle control at a user in a virtual environment. In order to improve performance, a static observer is used to estimate wind speed. This method will help in developing non-intrusive sensors for virtual environments.

\section{ACKNOWLEDGMENT}

The authors thank Mark Deaver, Charles Fisher, Richard Kirkman, Meredith Metzger and Pete Willemsen for being part of the TPAWT project.

\section{REFERENCES}

[1]J.M Hollerbach, "Locomotion interfaces," in Handbook of Virtual Environments: Design, Implementation, and Applications, e. K.M Stanley, Ed.: Lawrence Erlbaum Associates, Inc., 2002, pp. 239-254.

[2]R. Kirkman, M. Deaver, E. Pardyjak, and M.Metzger, "Sensitivity Analysis of a Three-Dimensional Wind Tunnel Design," in 2006 ASME Joint U.S.-European Fluids Engineering Summer Meeting, FEDSM, Miami, FL, 2006, p. pp. 10.
[3]S. D. Kulkarni, Minor, M.A., Deaver, M.W., and Pardyjak, E.R, "Output feedback control of wind display in a virtual environment," in Proc. IEEE Intl. Conf. Robotics and Automation, Rome, Italy, 2007, pp. 832 - 839.

[4]S. D. Kulkarni, Minor, M.A., Deaver, M.W., Pardyjak, E.R, and Hollerbach, J.M, "Steady Headwind Display with Conditional Angular Rate-Switching Control," in Proc. IEEE Intl. Conf. Robotics and Automation, Pasadena, California, 2008

[5]W. N. Dinh Huong Q., Song Chang, Kobayashi Akira, Hodges Larry F., "Evaluating the Importance of Multi-sensory Input on Memory and the Sense of Presence in Virtual Environments," Proceedings of the IEEE Virtual Reality, p. 222, 1999.

[6]Hassan.K.Khalil, Nonlinear Systems, 2002.

[7] S. S. Ravindran, "A reduced-order approach for optimal control of fluids using proper orthogonal decomposition," International Journal for Numerical Methods in Fluids, vol. 34, pp. 425-48, 2000/11/15 2000.

[8]A. Chatterjee, "An introduction to the proper orthogonal decomposition," Current Science, vol. 78, pp. 808-17, 2000/04/10 2000.

[9]P. D. Christofides and A. Armaou, "Global stabilization of the Kuramoto-Sivashinsky equation via distributed output feedback control," Systems and Control Letters, vol. 39, pp. 283-294, 2000.

[10] E. H. Dowell, K. C. Hall, and M. C. Romanowski, "Eigenmode analysis in unsteady aerodynamics: reduced order models," Applied Mechanics Reviews, vol. 50, pp. 371-385, 1997.

[11] M. Deaver, "Creating Wind Display for a Virtual Environment," in Mechanical Engineering. vol. Master of Science Salt Lake City: Utah, December 2007, p. 86.

[12] E. O. Doebelin, Measurement Systems : Application and Design, Third ed.: Mc-Graw Hill, 1983.

[13] Z. Hong, "Two-dimensional optimal sensor placement," IEEE Transactions on Systems, Man and Cybernetics, vol. 25, pp. 781-92, 1995.

[14] K. E. Gustafson, Vortex Methods and Vortex Motion. Philadelphia: SIAM, 1991.

[15] R. S. Sutton and A.G.Barto, Reinforcement Learning : An Introduction. Cambridge, MA: The MITpress, 1998.

[16] J. Peters and S. Schaal, "Policy Gradient Methods for Robotics," in Proceedings of the IEEE International Conference on Intelligent Robotics Systems (IROS), Beijing, China, 2006.

TABLE 3: OPEN LOOP FLOW MEASUREMENTS FOR THE VANE ANGLE

\begin{tabular}{|c|c|c|c|c|}
\hline Utotal & $\begin{array}{c}\mathrm{Y}_{\text {outlet }} \\
(\text { Mean })\end{array}$ & $\begin{array}{c}\mathrm{Y}_{\text {user }} \\
(\text { Mean })\end{array}$ & $\begin{array}{c}\mathrm{Y}_{\text {user }} / \mathrm{Y}_{\text {outlet }} \\
(\text { Speed Ratio })\end{array}$ & $\begin{array}{c}\text { Vane } \\
\text { Angle } \\
\left(\sim 30^{\circ}\right)\end{array}$ \\
\hline 7.0 & 2.87 & 1.90 & 0.66 & 34.50 \\
\hline 8.0 & 3.25 & 2.08 & 0.64 & 31.48 \\
\hline 10.0 & 3.98 & 2.46 & 0.62 & 32.49 \\
\hline 11.0 & 4.31 & 2.83 & 0.66 & 32.67 \\
\hline 12.0 & 4.66 & 3.08 & 0.66 & 32.81 \\
\hline 14.0 & 5.34 & 3.66 & 0.68 & 33.24 \\
\hline
\end{tabular}

